\title{
Wirtschaftsstrafverfahren: Entstehung eines neuartigen Verfahrentyps?
}

Eduard Güroff

\begin{abstract}
7 ahlreiche Anstrengungen des Gesetzgebers $\angle$ zur Bekämpfung der Wirtschaftskriminalität haben bislang nichts an erheblichen Implementationsdefiziten des Strafrechts ändern können. Der Versuch, wirtschaftliche Tätigkeit mit dem Strafrecht zu steuern, wurde daher schon frühzeitig als »Höhenflug mit Bauchlandung « bezeichnet. ${ }^{1}$ Einen besonderen Ausdruck finden diese Schwierigkeiten im Strafverfahren, was sich unter anderem darin äußert, dass Großverfahren regelmäßig Fälle von Wirtschaftskriminalität betreffen. ${ }^{2}$ Insofern spricht einiges für die Hypothese, dass die Leistungsfähigkeit des Prozessmodells der StPO hier an Grenzen stößt.
\end{abstract}

Diese Hypothese wird gestützt durch empirische Befunde aus einem Münsteraner Forschungsprojekt zur Verbreitung und Kontrolle der Wirtschaftskriminalität im Zusammenhang mit der Privatisierung ehemals volkseigener Betriebe durch die Treuhandanstalt. ${ }^{3}$ Die Befunde deuten darauf hin, dass sich die Praxis von Wirtschaftsstrafverfahren weitgehend von dem Prozessmodell der StPO entfernt hat und die Beteiligten zunehmend auf der Grundlage eines bislang erst in Umrissen erkennbaren Verfahrenstyps operieren. Das Forschungsprojekt zielt darauf $a b$, nähere Erkenntnisse darüber zu gewinnen, ob und unter welchen Bedingungen eine strafrechtliche Kontrolle von Wirtschaftskriminalität möglich ist. Im Rahmen einer explorativ konzipierten qualitativen Untersuchung wurden Privatisierungsvorgänge mit strafrechtlicher Relevanz untersucht. Strafrechtlich relevante Privatisierungsvorgänge waren dabei solche, bei denen es zur Aufnahme von Ermittlungen kam, ohne dass diese Vorgänge zwingend zu Verurteilungen führen mussten. Nach einer Analyse der Ermittlungsakten und - soweit ergangen - von Strafurteilen wurden Experteninterviews mit den an diesen Privatisierungsvorgängen und ihrer strafrechtlichen Kontrolle in unterschiedlichen Rollen beteiligten Personen geführt: Beschuldigten, Rechtsanwälten, Richtern und Staatsanwälten.

\section{Das traditionelle Prozessmodell}

Mit der Einschränkung, dass Prozessmodelle selten in Reinform auftreten, kann man das Prozessmodell der StPO als staatlich betriebenen Anklageprozess mit einer gerichtlichen Amtsaufklärung charakterisieren. ${ }^{4}$ Es steht als reformierter Strafprozess in einem Gegensatz sowohl zum Inquisitionsprozess, bei dem das Recht zur Anklage und zum Urteil in einer Institution vereinigt sind, als auch zu dem Prozessmodell des US-amerikanischen Strafrechts,

das zum einen durch die Parteistellung von Staatsanwaltschaft und Verteidigung bzw. Beschuldigtem sowie zum anderen durch die Dispositionsmaxime und den Beibringungsgrundsatz geprägt ist, so dass die über den Verfahrensgegenstand verfügungsberechtigten Parteien verpflichtet werden, die Beweismittel dem erkennenden Gericht vorzuführen. ${ }^{5}$

Demgegenüber zeichnet sich die StPO hinsichtlich des Ermittlungsverfahrens und der Anklageerhebung durch das staatliche Strafverfolgungs- und Anklagemonopol, das normative Objektivitätspostulat ( $\$ 160$ Abs. 2 StPO) und jedenfalls in ihrer ursprünglichen Fassung von 1877 durch das Legalitätsprinzip aus ( $\$ 152$ Abs. 2 StPO), das die Staatsanwaltschaft zur Strafverfolgung und Anklage verpflichtet. ${ }^{6}$ Nach Anklageerhebung sind für das gerichtliche Verfahren die Grundsätze der Mündlichkeit (§§ 261, 264 StPO) und Unmittelbarkeit ( $\$ 250$ StPO) sowie der Öffentlichkeit (§ 169 GVG) kennzeichnend, ferner der Grundsatz der freien Beweiswürdigung (§ 261 StPO) und schließlich die Verpflichtung auf einen materiellen Wahrheitsbegriff (§§ 244 Abs. 2; 261 StPO).

Aufs Ganze besehen kann dieses Prozessmodell als Ausdruck des traditionell universalen Geltungsanspruchs des Strafrechts verstanden werden, der sich aus zwei Fundamentalbeziehungen des Modells ergibt, die zum einen im Hinblick auf die grundlegende straftheoretischen Konzeption und zum anderen im Hinblick auf die Stellung des Strafrechts innerhalb der Gesellschaft bestehen:

Zumindest in seiner ursprünglichen Form zielte das Prozessmodell der StPO auf die vollständige Aufklärung des Sachverhalts und die gleiche Vergeltung aller gleich Schuldigen, indem sich richterliche Organe darum bemühen sollten, durch intensive Sachverhaltsaufklärung unter Heranziehung aller verfügbaren Beweismittel eine möglichst verlässliche Tatsachengrundlage für die abschließende Entscheidung des Gerichts herzustellen. ${ }^{7}$ Diese Orientierung an Wahrheit und Gerechtigkeit ${ }^{8}$ bedingte eine deutliche Affinität dieses Prozessmodells zur klassischen Straftheorie der Vergeltung, die den Sinn der Strafe darin sieht, durch die Zufügung eines Strafübels das durch die Tat verursachte Unrecht zu tilgen. ${ }^{9}$ Vergeltung kann aber nur erreicht werden, wenn die Schuld des Täters gemäß dem materiellen Wahrheitsbegriff aufgrund vollständiger Sachverhaltsaufklärung ermittelt wird und zugleich über das Legalitätsprinzip gewährleistet wird, dass wirklich »jedermann das widerfahre, was seine Taten wert sind $« .{ }^{10}$ Weniger deutlich als die Beziehung zwischen dem traditionellen Prozessmodell und der grundlegenden straftheoretischen Konzeption ist die Beziehung zwischen den im materiellen Wahrheitsbegriff sowie im Legalitätsprinzip

verkörperten strafprozessualen Zielsetzungen von Wahrheit und Gerechtigkeit und dem zugrunde liegenden Strafrechtsverständnis ausgeprägt. Letztlich basierte das Modell auf einem autoritativen Strafrechtsverständnis, das von einem klaren Über-/Unterordnungsverhältnis zwischen dem Strafrecht und den Strafrechtsunterworfenen ausging und gerade deshalb strafprozessuale Zugriffe auf die Lebenswelt der Strafrechtsunterworfenen als möglich erachtete. ${ }^{11}$ Nur ein Verhältnis der Über-/Unterordnung bietet die faktische Gewähr, dass der strafende Staat einen umfassenden Zugriff auf diese Lebensbereiche erhält und auf der Grundlage vollständiger Sachverhaltsermittlung in gleichmäßiger Weise Schuld vergilt.

\section{Der Erosion der Grundlagen des traditionellen Prozessmodells}

Auch wenn die StPO seit 1877 zahlreiche und auch gravierende Modifikationen erfahren hat, hat das traditionelle Prozessmodell gleichwohl die Grundstruktur des Strafprozesses vorgegeben und eignet sich als Vergleichsmaßstab für die aktuelle Verfahrenspraxis. Deren Entfernung von dem traditionellen Prozessmodell ist darauf zurückzuführen, dass diese beiden das Modell tragenden Fundamentalbeziehungen brüchig geworden sind. An die Stelle des traditionellen Vergeltungskonzeptes ist trotz § 46 Abs. 1 Satz 1 StGB ein zunehmend funktionales Strafrechtsverständnis getreten. Zudem ist das traditionelle Über-/Unterordnungsverhältnis jedenfalls im Bereich der Wirtschaftskriminalität durch die gesellschaftliche Realität überholt worden. 
Die RStPO von 1877 diente der Umsetzung eines dem ultima-ratio-Gedanken verpflichteten materiellen Strafrechts, das auf der Grundlage des Vergeltungsgedankens allein das »ethische Minimum « innerhalb der Gesellschaft und eine reaktive Krisenbewältigung im Einzelfall gewährleisten sollte. ${ }^{12}$ Im Gegensatz dazu verbindet sich mit dem modernen materiellen Wirtschaftsstrafrecht die Hoffnung auf eine planvolle Gestaltung gesellschaftlicher Ordnung, in deren Dienst das Strafrecht gestellt wird. ${ }^{13}$ Das funktionale Strafrechtsverständnis findet Ausdruck in der Ablösung des ursprünglichen Vergeltungs- durch ein Präventionskonzept, ${ }^{14}$ das auf die Unterbindung strafrechtswidrigen Verhaltens mit möglichst geringem personellem und institutionellem Aufwand gerichtet sein muss. ${ }^{15} \mathrm{Zu}$ seiner Absicherung ist die vollständige und gleichmäßige Durchsetzung des materiellen Strafrechts nicht erforderlich, sondern es genügt die Aufrechterhaltung eines wahrnehmbaren und sich im Einzelfall realisierenden Sanktionierungsrisikos. ${ }^{16}$ Die dem traditionellen Prozessmodell zugrunde liegende

Vorstellung der gleichen Vergeltung aller gleich Schuldigen wird damit durch die Idee der Selektion ersetzt. Dementsprechend äußerte ein Interviewpartner:

"Als Wirtschaftsstaatsanwalt muss man wissen, dass man für jeden intensiv verfolgten Täter einen anderen straflos oder mit einer unverdient milden Bewährungsstrafe davonkommen lässt" (1-2-1 79/102).

Aber selbst innerhalb eines einzelnen Strafverfahrens ist es angesichts der Komplexität und des Umfangs des Verfahrensstoffes unmöglich, eine vollständige Sachverhaltsaufklärung und Durchsetzung des staatlichen Strafanspruchs zu erreichen. Ein Staatsanwalt schilderte die Bedeutung des Selektionsparadigmas:

"Massen bewältigt man mit sMut zur Lücker, wobei man nach dem sPrinzip Hoffnung vorgeht: die Tatsache, dass andere auch noch einmal in den Akten herumgeblättert haben, veranlasst zu der optimistischen Hoffnung, dass nichts Relevantes übersehen wurde. [...] Man kann hier nicht alles Blatt für Blatt in epischer Breite saufdröseln««(1-4-1 788/792; 7344/7430).

Die Kehrseite dieser durch den selektiven Einsatz des Strafverfahrens erzielten »Präventionsökonomie " besteht darin, dass die Durchsetzung des materiellen Rechts auf der Ebene des Strafverfahrens zunehmend als willkürlich wahrgenommen wird. Ein Strafverteidiger kritisierte:

»Gerade die Verfolgung von Wirtschaftskriminalität läuft sehr willkürlich $a b$. [...] Die StPO ist der >Mülleimer des materiellen Strafrechts ge- worden $[\ldots] \ll \quad(9-2-1 \quad 272 / 339 ; \quad 2452 / 2555$; 2753/2974; 3234/3325).

Das traditionelle Verständnis eines Über-/ Unterordnungsverhältnisses geht an der Wirklichkeit von Wirtschaftsstrafverfahren vorbei, weil wirtschaftliche Akteure regelmäßig aufgrund ihrer ökonomischen Potenz in der Lage sind, dem durch die Strafjustiz konkretisierten staatlichen Strafanspruch wirkungsvoll entgegenzutreten. Die formalen Befugnisse der strafrechtlichen Kontrollinstanzen werden damit in materieller Hinsicht ausgeglichen, was ein Interviewpartner aus der Staatsanwaltschaft folgendermaßen beschrieb:

"Die Staatsanwaltschaft ist personell und sachlich im Nachteil, teilweise ist sie auch überfordert«(1-9-1 1685/1705).

Eine besondere Bedeutung kommt der Beauftragung von Gutachtern zu, die die den Gegenstand des Strafverfahrens bildenden wirtschaftlichen Handlungen unter jeweils spezifischen fachlichen Gesichtspunkten bewerten und insoweit in erheblicher Weise den Gang des Verfahrens bestimmen. Ein Verteidiger erklärte:

»Wenn man in einer Wirtschaftsstrafsache vernünftig verteidigt werden will, benötigt man Geld, da man sich Sachverstand einkaufen muss [...]« (9-4-1 1390/1570).

Die Erosion des traditionellen Über-/Unterordnungsverhältnisses zeigt sich insbesondere dann, wenn es um strafrechtlich relevantes Verhalten in Unternehmenszusammenhängen geht, da ein Unternehmen in der Regel über größere finanzielle Mittel als Individualpersonen verfügt. Mit Blick auf ein untersuchtes Verfahren erläuterte ein Strafverteidiger:

"Das Unternehmen war natürlich ein >Traummandants, da alle zur Verteidigung erforderlichen Maßnahmen wie Aktenrecherche oder Gutachten gezahlt worden sind. Der normale Mandant geht irgendwann in die Knie, wenn mehr als 500 Anwaltsstunden auf ihn zukommen $\lll(9-3-3$ 1200/1251).

Angesichts des Potenzials, dem strafrechtlichen Zugriff wirkungsvoll entgegenzutreten, resümierte ein Staatsanwalt:

»Die Bekämpfung von Wirtschaftskriminalität ist durch ein erhebliches Ressourcenungleichgewicht geprägt. Wird ein Konzern massiv von den Strafverfolgungsorganen ins Visier genommen, so wehrt er sich quantitativ - etwa über die Benennung zahlreicher Zeugen - oder qualitativ - etwa durch Einholung von Fachgutachten - in einer Weise, dass die Staatsanwaltschaft dagegen nicht mehr angehen kann. [...] Ein Konzern lässt dann sanze Batterien auffahren, [...], Geld spielt keine Rolle mehr und die Staatsanwalt- schaft gerät in ein nicht mehr handhabbares Verfahren « (1-3-2 A. 3270/3282).

\section{Die Verfahrenspraxis}

Angesichts der Brüchigkeit der dem traditionellen Prozessmodell zugrunde liegenden Fundamentalbeziehungen musste das traditionelle Prozessmodell der StPO an Grenzen stoßen. Dieser Befund wird deutlich anhand einer weit verbreiteten Anwendung nachträglich in die StPO aufgenommener flexibler Erledigungsinstrumente, einer zumindest faktischen Aufgabe des materiellen Wahrheitsbegriffs und einer Schwerpunktverschiebung zwischen den verschiedenen Verfahrensabschnitten.

Die Ablösung des Vergeltungskonzepts durch ein Präventionskonzept und die Ablösung des Über-/Unterordnungsverhältnisses führen gleichermaßen dazu, dass an die Stelle gleichmäßiger Strafrechtsanwendung das Selektionsparadigma tritt. Wenn die gleichmäßige und vollständige Durchsetzung des staatlichen Strafverfahrens zur Aufrechterhaltung des Präventionsniveaus weder nötig noch möglich ist, muss dem hieraus entstehenden Bedürfnis nach selektiver Strafrechtsanwendung Rechnung getragen werden. Dies geschah durch die Aufnahme flexibler - und konsensualer - Erledigungsinstrumente wie dem Strafbefehlsverfahren nach $\S \S 407$ ff. StPO und den Opportunitätsvorschriften nach $\S \S 153$ ff. StPO in die StPO, die die Akzeptanz des Strafbefehls durch den Beschuldigten oder seine Zustimmung des Beschuldigten voraussetzen. ${ }^{17}$ Obwohl das Strafverfahren hier nicht mit einem Strafurteil endet, gelingt es mit diesen flexiblen Erledigungsinstrumenten ein gewisses Sanktionierungsrisiko zu entfalten, das gleichermaßen zur Aufrechterhaltung des angestrebten Präventionsniveaus geeignet ist wie die vollständige Durchsetzung des staatlichen Strafanspruchs im Rahmen eines kontradiktorisch geführten Verfahrens. Ein Verteidiger:

"Es ist eine befriedigende Regelung wirtschaftsstrafrechtlicher Fälle, wenn man zeigt, dass die strafrechtliche Drohung gewirkt hat, eine Haftstrafe aber etwa durch [...] Auflagen [nach $\S 153$ a StPO, Anm. Verf.] verhindert wird. Hier hat man den Nachweis, dass das Strafrecht wirkt, ohne dass man Leute einsperren muss " (9-3-3 5649/5686).

Die praktische Bedeutung dieser flexiblen Erledigungsinstrumente ist umso größer, als die gleichmäßige und vollständige Durchsetzung des staatlichen Strafanspruchs im Bereich der Wirtschaftskriminalität ohnehin nicht oder allenfalls bei erheblichen Folgekosten möglich ist. Ein Staatsanwalt erklärte dementsprechend mit Blick auf die drohende instrumentelle Überforderung der Strafjustiz: 
»Von $\S 153$ a StPO wird vor allem aus Ressourcengründen Gebrauch gemacht. Es handelt sich richtiggehend um ein Einknicken $<$ vor den Prozessen. [...] (1-4-1 7582/7592; 8389/8399).

Da sich die Schere zwischen der dem Strafrecht gestellten Präventionsaufgabe und der realen Bearbeitungskapazität der Strafjustiz immer weiter öffnet, überrascht es nicht, dass die flexiblen Erledigungsinstrumente in der praktischen Anwendung eine expansive Tendenz aufweisen. Diese äußert sich darin, dass die tatbestandlichen Voraussetzungen der Opportunitätsvorschriften - etwa die einer Verfahrenseinstellung nicht entgegenstehende »Schwere der Schuld « in § 153a StPO - gerade in Wirtschaftsstrafverfahren sehr extensiv interpretiert werden. So erklärte ein Staatsanwalt:

"Früher lag die Grenze für Verfahrenseinstellungen bei DM 150, heutzutage werden demgegenüber auch Millionenverfahren über $\S 153$ a StPO eingestellt «(1-3-1 2778/2800).

In diese Entwicklung fügt sich das Phänomen der verfahrenserledigenden Urteilsabsprachen ein, die sich aus der Praxis heraus entwickelt haben und - nachdem sie lange im Verborgenen praktiziert wurden $-{ }^{18}$ nunmehr höchstrichterlich gebilligt werden. ${ }^{19}$ Das Verfahren endet hier mit einem für den Angeklagten im Vergleich zu einem kontradiktorisch geführten Verfahren regelmäßig günstigeren Urteil, dessen tatsächliche und rechtliche Grundlagen letztlich auf einem Konsens zwischen den Verfahrensbeteiligten, nicht aber einer vollständigen Sachverhaltsermittlung und Durchsetzung des staatlichen Strafanspruchs durch die Strafjustiz beruhen. Ein Staatsanwalt berichtete, dass im Rahmen eines Strafverfahrens Absprachen getroffen worden seien, um ergebnisorientiert arbeiten zu können und die Staatsanwaltschaft somit vor einer instrumentellen Überforderung zu schützen:

\section{"Hätte man alles aufarbeiten wollen, hätte das Verfahren fünf Jahre gedauert" (1-2-1 1600/1641).}

Ähnlich wie bei den Einstellungsvorschriften zeigt sich auch im Bereich der Urteilsabsprachen, dass von den flexiblen Erledigungsinstrumenten offenbar großer Gebrauch gemacht wird. Insbesondere werden die diesbezüglichen höchstrichterlichen Vorgaben von der Praxis wohl häufig ignoriert, was gerade für die auch im Kontext von Absprachen geltenden Grundsätze eines schuldangemessenen Strafens und der Öffentlichkeit sowie das Verbot der Vereinbarung eines Rechtsmittelverzichts gilt. ${ }^{20}$ Ein befragter Strafverteidiger schilderte:

»Unter einem, Deak verstehe ich, wenn der Staatsanwalt sagt ,Ich habe hier 300 Millionen Schaden, der sich aus vier Gruppen zusammen- setzt und die Verteidigung erwidert , Gut, pass mal auf, nimm mal drei weg, dann haben wir noch 40 Millionen. Was soll das kosten? Drei Jahre? Dann gehen wir in einen Prozess, morgens um acht, die Presse schläft noch und alle nicken heftig. Rechtsmittelverzicht, den hinterlegt, sozusagen zu treuen Händen " (9-4-1 1246/1386; 1214/1244).

Über die mit den flexiblen Erledigungsinstrumenten ohnehin zwangsläufig verbundene Minderung der strafprozessualen Sachverhaltsaufklärung hinaus zeichnet sich die Praxis von Wirtschaftsstrafverfahren durch eine faktische Verabschiedung des materiellen Wahrheitsbegriffs aus. Diese Entwicklung erfolgt vor dem Hintergrund einer allgemein in der Strafrechtswissenschaft beobachtbaren »Entzauberung « des materiellen Wahrheitsbegriffs. ${ }^{21}$ Diese reicht von Hinweisen darauf, dass der strafprozessualen Wahrheit allein schon wegen ihres Bezuges auf das materielle Strafrecht eine »Finalstruktur « innewohne ${ }^{22}$ und je nach Art der strafprozessualen Entscheidung »diverse Wahrheiten « existierten, ${ }^{23}$ bis hin zu der Überzeugung, dass die Wahrheit des Strafprozesses nie die vollständige und zutreffende Rekonstruktion eines den Gegenstand des Strafverfahrens bildenden Wirklichkeitsausschnitts ist, sondern stattdessen ein in einem formalisierten Verfahren durch die Interaktion verschiedener Verfahrensbeteiligter erzeugtes Konstrukt. ${ }^{24}$ Im Kontext von Wirtschaftsstrafverfahren potenziert sich das Problem und die strafprozessuale Rekonstruktion des den Verfahrensgegenstand bildenden Wirklichkeitsausschnitts erscheint hier schon aufgrund der Komplexität und des Umfangs der dem Verfahren zugrunde liegenden Tatvorwürfe als vollends unrealisierbare Zielvorgabe. ${ }^{25}$ So schilderte ein befragter Richter unter Bezug auf ein Strafverfahren:

"Allein für das Aktenmaterial [das als Beweismaterial diente, Anm. Verf.] musste eine Turnhalle angemietet werden « (10-9-2 1291/1386).

Angesichts dieser Schwierigkeiten erscheinen die das Strafverfahren prägenden Grundsätze zur Rekonstruktion des dem Strafverfahren zugrunde liegenden Wirklichkeitsausschnitts für Wirtschaftsstrafverfahren als inadäquat. ${ }^{26}$ Insbesondere die für das Hauptverfahren geltenden Grundsätze der Unmittelbarkeit und Mündlichkeit stellen die Strafjustiz vor massive Probleme, zu denen ein Staatsanwalt ausführte:

"Als die Vorschriften über die Verlesung von Urkunden in die StPO aufgenommen worden sind, hat man an drei oder vier Blättchen und eine Rechnung gedacht, aber nicht an tausend Ordner $\ll(1-3-13590 / 3640)$.

Diese Probleme führen dazu, dass die Ermittlung der verfahrensrelevanten Tatsachen nicht allein durch die Strafjustiz erfolgt, sondern Ver- teidigung und Beschuldigter oftmals frühzeitig in die Sachaufklärung einbezogen werden. Ein Wirtschaftskriminalist erläuterte:

»In Wirtschaftsstrafverfahren müssen Sie Ihren Gegenüber überzeugen und motivieren, halbwegs mitzuarbeiten, da die Sache kompliziert ist und der Gegenüber notwendigerweise mehr weiß als man selbst. Denn was soll ich denn fragen? Ich muss doch erst einmal selbst fragefähig werden " (2-3-1 2261/2272).

Aus Sicht der Verteidigung und des Beschuldigten dienen derartige Formen der Kooperation nicht nur dazu, strafprozessuale Zwangsmaßnahmen $\mathrm{zu}$ vermeiden, sondern sie eröffnen darüber hinaus die Möglichkeit, Einfluss auf die Festlegung des entscheidungsrelevanten Sachverhalts zu gewinnen. In der Verfahrenswirklichkeit wird damit die materielle Wahrheit des traditionellen Prozessmodells gewissermaßen durch eine »konsentierte Wahrheit« abgelöst, was ein Strafverteidiger folgendermaßen beschrieb:

"Gerade bei komplexen Verfahren dient ein kooperativer Verhandlungsstil dazu, sich mit der Staatsanwaltschaft eine gemeinsame Tatsachengrundlage zu erarbeiten, auf der man dann anschließend rechtlich argumentieren kann « (9-3-2 925/954).

Diese Gesamtentwicklung führt notwendig zu einer Schwerpunktverschiebung zwischen den einzelnen Verfahrensabschnitten. Während das gerichtliche Hauptverfahren traditionell den Verfahrensschwerpunkt bildete, schwindet in Wirtschaftsstrafverfahren dessen Bedeutung zu Lasten des Ermittlungsverfahrens. ${ }^{27}$ Damit ergibt sich die in gewissem Sinne paradoxe Situation, dass das durch die Grundsätze der Unmittelbarkeit und Mündlichkeit geprägte Hauptverfahren zur prozessualen Aufbereitung des dem Strafverfahren zugrunde liegenden Sachverhalts weniger geeignet ist als das Ermittlungsverfahren. Ein Strafverteidiger:

"Die Kompliziertheit der wirtschaftlichen Zu-
sammenhänge führt dazu, dass man viele Dinge,
die man im Ermittlungsverfahren in Ruhe be-
sprechen und vortragen kann, im Gerichtssaal
gar nicht kommunizieren kann. Wir wissen ja
um die beschränkten Möglichkeiten, die man im
Saal hat" (9-2-2 209/229; 956/1002; 168/187).

Eine solche Entwicklung hat notwendig Konsequenzen für das Verhältnis der Verfahrensbeteiligten zueinander. Insbesondere verbindet sich mit ihr eine zu Lasten des Gerichts gehende Aufwertung der Staatsanwaltschaft. Vor diesem Hintergrund weisen Wirtschaftsstrafverfahren zunehmend Anklänge an einen Parteiprozess auf. ${ }^{28}$ Ein Richter schilderte: 
"Heutzutage hat sich die Position des Gerichts im Vergleich zu der der Staatsanwaltschaft und der Verteidigung geschwächt, die vieles unter sich im Ermittlungsverfahren ausmachen und für die Hauptverhandlung die Richtung vorgeben " (10-92 1291/1386).

Diese Entwicklung drückt sich auch in einem gewandelten Rollenverständnis aus. So ist für Wirtschaftsstrafverfahren das Auftreten eines Verteidigertypus kennzeichnend, der sich nicht nur durch besondere Sachkunde und Seriosität, sondern auch durch seine besondere Verpflichtung auf das Mandanteninteresse auszeichnet. ${ }^{29}$ Ein Strafverteidiger erklärte insoweit unter Bezugnahme auf $\S 1$ BRAO: ${ }^{30}$

"Auf den Titel `Organ der Rechtspflege gebe ich gar nichts. Im Zweifel verstehe ich mich als Interessenvertreter des Mandanten - dies aber mit Grenzen«(9-4-1 3283/3361).

Diese Vorverlagerung des Verfahrensschwerpunktes liegt durchaus im Interesse von Verteidigung und Beschuldigtem. Insbesondere aus Sicht der Verteidigung bzw. des Beschuldigten besteht regelmäßig ein Interesse an der Vermeidung einer lang andauernden Hauptverhandlung, da die damit verbundene Öffentlichkeitswirkung erhebliche negative Folgewirkungen im gesellschaftlichen und wirtschaftlichen Bereich mit sich bringt. Der ursprünglich dem Interesse des Beschuldigten dienende Öffentlichkeitsgrundsatz weist deshalb gerade in Wirtschaftsstrafverfahren ein erhebliches Bedrohungspotential auf. ${ }^{31}$ Ein Strafverteidiger erläuterte:

"Die Klientel will alles, nur keinen Prozess. [...] Das Schlimmste in einer Wirtschaftssache ist eine Hauptverhandlung, in der vom Verdienst bis zu Amouren oder Peinlichkeiten alles zur Sprache kommt und Partei und Freunde im Sitzungssaal sitzen und feixen [...] (9-4-1 1029/1119).

Auch mit Blick auf eine zu erwartende Sanktion besteht aus Sicht von Verteidigung und Beschuldigtem ein erhebliches Interesse, den Prozess vor einer kontradiktorisch geführten Hauptverhandlung zu beenden, womit freilich die faktische Sanktionsgewalt zunehmend vom Gericht auf die Staatsanwaltschaft verlagert wird. Ein Verteidiger schilderte:

"Bei Gericht sind die sPreiser anders. Der Witz an einer Wirtschaftsstrafsache besteht deshalb darin, das `Ding vor der Hauptverhandlung zuzumachen. Wenn es erst einmal in die Hände des Gerichts kommt, wird es schwierig. [...] Demgegenüber kann man bei der Staatsanwaltschaft auch wirklich große Sachen mit Strafbefehl szumachen« (9-4-1 2064/2211; 2237/2357).

\section{Auf dem Weg zu einem neuartigen Verfahrenstyp?}

Die empirischen Befunde zeigen, dass sich die Verfahrenspraxis von dem traditionell der StPO zugrunde liegenden Prozessmodell entfernt hat. Zusammenfassend lässt sich der neu entstehende Verfahrenstyp weniger als Amts-, sondern als Parteiprozess mit Staatsanwaltschaft und Verteidigung bzw. Beschuldigtem als Antagonisten beschreiben, die schon im Ermittlungsverfahren wesentlichen Einfluss auf den Verfahrensausgang nehmen. Die Wahrheit dieses Verfahrenstyps ist keine materielle, sondern eine solche, auf die sich die Parteien verständigen. Eine Verständigung erfolgt auch hinsichtlich des Verfahrensergebnisses, da ein Strafurteil nach kontradiktorisch geführtem Verfahren die Ausnahme ist, während flexible Erledigungsformen oder Urteilsabsprachen den Regelfall bilden. Die das traditionelle Prozessmodell kennzeichnende Idee einer vollständigen Aufklärung des dem Strafverfahren zugrunde liegenden Sachverhalts und der gleichmäßigen Vergeltung aller gleich Schuldigen zugunsten eines Verfahrenstyps aufgegeben, der sich durch Selektivität und Konsens auszeichnet und sich insoweit auch nicht mehr als Ausdruck eines universalen strafrechtlichen Geltungsanspruchs begreifen lässt.

Mit Blick auf die Brüchigkeit der beiden Fundamentalbeziehungen des traditionellen Prozessmodells spricht wenig dafür, dass eine Rückkehr zum Status quo ante möglich ist. Eine angesichts leerer öffentlicher Kassen ohnehin unwahrscheinliche Vermehrung von personellen und sachlichen Ressourcen der Strafjustiz letztlich also "more of the same - würde an der grundsätzlich strukturellen Problematik wenig ändern. ${ }^{32}$ Abzulehnen ist der Vorschlag, die auf das Verfahren bezogenen »Obstruktionsmöglichkeiten« von Verteidigung und Beschuldigtem zu verringern, ${ }^{33}$ da erstens die Grenzziehung zwischen illegitimer Verfahrensobstruktion und legitimer Interessenwahrnehmung kaum möglich ist und zweitens ein $\mathrm{Ab}$ bau rechtsstaatlicher Verfahrensgarantien das rechtsstaatliche Prozessmodell der StPO nur auf andere Weise strukturell beeinträchtigenden würde. ${ }^{34}$ Eine materiellrechtliche Entkriminalisierung wäre grundsätzlich zu begrüßen, ${ }^{35} \mathrm{er}$ scheint aber aufgrund des im Zweifel eher auf weitere Kriminalisierungen gerichteten rechtspolitischen Klimas unrealistisch. Im Übrigen mutet es seltsam an, ausgerechnet in der Rückkehr zu einem reinen - letztlich metaphysischen - Vergeltungskonzept und der Ablehnung jeder gesellschaftlichen Funktion des Strafrechts einen Fortschritt zu sehen. ${ }^{36}$ Man wird sich also auf den aus der Verfahrenspraxis heraus entstandenen neuartigen Verfahrenstypus einzustellen haben, wobei bislang noch nicht deutlich ist, in welche Richtung sich die Verfahrenspraxis entwickelt.
Die Gefahren eines durch Selektivität und Konsens gekennzeichneten Verfahrenstyps sind nicht zu verkennen:

Während aufgrund des universalen Geltungsanspruchs des Strafrechts in dem traditionellen Prozessmodell das Strafverfolgungs- und Verurteilungsrisiko zumindest der Idee nach gleichmäßig verteilt sein sollte, wird die Ungleichmäßigkeit der Strafrechtsanwendung auf der Basis des Selektionsparadigmas zum System. Gerade weil der neuartige Verfahrenstypus nicht gleichermaßen für sämtliche Strafverfahren gilt, sondern vornehmlich in Wirtschaftsstrafverfahren praktiziert wird, kann der Eindruck einer »Bevorzugung von Wohlstandskriminellen « entstehen. ${ }^{37}$ Die Gleichmäßigkeit der Strafrechtsanwendung bildet jedoch eine wesentliche Legitimationsbedingung des Strafrechts. Auch das Konsensparadigma stellt sich als problematisch dar. Trotz des universalen strafrechtlichen Geltungsanspruchs diente auf der Basis des traditionellen Prozessmodells auch die Förmlichkeit des Verfahrens dem Schutz des als Prozesssubjekt begriffenen Beschuldigten, ${ }^{38}$ während keineswegs ausgemacht ist, dass die Konsensidee des neuartigen Verfahrenstyps die Autonomie des Beschuldigten gewährleistet und dieser nicht etwa zu einem "Konsens « gezwungen ${ }^{39}$ und deshalb zum Unterlegenen in einem nicht mehr in den Kategorien des Rechts, sondern nur noch nach Machtkategorien gelösten Konflikt degradiert wird. ${ }^{40}$ Auch hier droht eine wesentliche Legitimationsbedingung des Strafrechts zu entfallen.

Insgesamt betrachtet ist die Gefahr nicht von der Hand zu weisen, dass die Entwicklung des neuartigen Verfahrenstyps dazu führt, dass dem Strafrecht eine bislang ihm exklusiv zukommende gesamtgesellschaftliche Funktion abhanden kommt, die darin besteht, verbindlich die Grenze zwischen Recht und Unrecht festzulegen und sich dabei der dramatisierenden Szenerie des Strafprozesses zu bedienen. Am Ende stellt sich die Frage: Ist das, was im Bereich der Wirtschaftsstrafverfahren beobachtbar ist, noch Strafrecht oder zumindest die dem Strafrecht gemäße prozessuale Aufarbeitung eines Tatvorwurfs?

Diese Gefahren müssen sich nicht zwangsläufig realisieren, zumal auch im außerstrafrechtlichen öffentlichen Recht zunehmend kooperative oder konsensuale Gestaltungsformen gegenüber autoritativem staatlichem Handeln an Boden gewinnen. ${ }^{41}$ Die entscheidende Frage ist daher, ob es gelingt, die das traditionelle Prozessmodell prägenden Gerechtigkeitsideen von Wahrheit und Gerechtigkeit durch andere Gerechtigkeitsparameter zu ersetzen. Da sich ein durch Selektivität und Konsens geprägtes Prozessmodell nicht mehr darauf stützen lässt, dass die am Ende des Strafverfahrens stehende Entscheidung auf einer vollständigen Ermitt- 
lung der Wahrheit beruht und gerade deswegen "gerecht « ist, müssen an die Stelle materialer zunehmend formelle Gerechtigkeitskriterien treten. Insoweit könnte etwa die - prozedurale Idee von Fairness in Betracht kommen. ${ }^{42}$ Die Legitimität des Verfahrens hinge dann von der Fairness der jeweiligen Selektionsentscheidung und des jeweiligen Konsenses ab.

Bezogen auf das einzelne Strafverfahren müssten Vorkehrungen getroffen werden, die die Fairness der dort erfolgenden Selektionsentscheidungen und Konsensfindungen gewährleisten. Insbesondere wäre die Autonomie des Beschuldigten in einer den Strukturen des neuartigen Verfahrenstyps entsprechenden Weise sicherzustellen, ${ }^{43}$ was nicht nur im Rahmen der jeweiligen Erledigungsform des Strafverfahrens, sondern auch für die Generierung konsentierter Wahrheit gilt und Anlass dazu gibt, den Beschuldigten, sofern dieser bereit dazu ist, in den Prozess der Wahrheitsfindung einzubeziehen, auch wenn ihn aufgrund des Selbstbelastungsverbots insoweit natürlich keine Mitwirkungspflicht treffen kann. Die

Vorverlagerung des Verfahrensschwerpunktes in das Ermittlungsverfahren legt es überdies nahe, Beschuldigtem und Verteidigung hier größere Partizipationsmöglichkeiten zuzugestehen. Schon zum Schutz der Stellung des Beschuldigten als Prozesssubjekt müsste freilich stets eine Rückkehrmöglichkeit zum kontradiktorischen Normalverfahren erhalten bleiben.

Schwieriger wäre die mit der Koexistenz von kontradiktorischem traditionellen Strafverfahren und dem neuen Verfahrenstyp gegebene Binnendifferenzierung des Strafrechts und seines Verfahrenssystems unter Fairnessgesichtspunkten zu legitimieren. ${ }^{44}$ Hier käme es darauf an, einerseits der Strafjustiz Freiräume für Schwerpunktsetzungen in der Strafverfolgung zu ermöglichen, andererseits aber gesamtgesellschaftlich das Strafverfolgungs- und Verurteilungsrisiko fair zu verteilen. Dies könnte Anlass zu Überlegungen sein, über einen »übergreifenden Einsatzplan « nachzudenken, der den normativen Sinn des Legalitätsprinzips - also Gleichheit und Gerechtigkeit - wahrt und zugleich den nur an Schwerpunkten orientierten Zugriff legitimiert. ${ }^{45}$

Hans Theile ist wissenschaftlicher Assistent am Institut für Kriminalwissenschaften der Universität Münster

\section{Fußnoten:}

1 Liebl, KrimJ 1986, 50 (56).

2 Vgl. etwa Tiedemann, JuS 1989, 689 (698).

3 Das von der DFG geförderte Projekt wird an der Universität Münster unter der Leitung von Prof.
Dr. Klaus Boers und Prof.in Dr. Ursula Nelles durchgeführt. Zu dem Forschungsprojekt siehe Boers, MSchrK 2001, 335 (343 ff.); Boers/Theile/Karliczek, KZfSS Sonderheft Nr. 43/2003, 469 (472 f.); Karliczek, in: Kriminologische Erkundungen, hrsg.: Karliczek (2004), 210 (211 ff.).

4 Eser, ZStW 104 (1992), 360 (363 f.); Löwe/Rosenberg - Rieß, Einl. Abschn. F Rn. 9 f.

5 Löwe/Rosenberg - Rieß, Einl. Abschn. F Rn. 9 f.

6 a.a.O.

7 Weigend, NStZ 1990, 774 (780 f.); ders., ZStW 113 (2001), 271 (271).

8 Weigend, ZStW 113 (2001), 271 (271).

9 Vgl. Eser, ZStW 104 (1992), 360 (363 f.); Krauß, FS Schaffstein (1975), 411 (424 ff.); Weigend, JZ 1990, 774 (780 f.).

10 Kant, Metaphysik der Sitten, Rechtslehre, 2. Teil, 1. Abschnitt E.

11 Eser, ZStW 104 (1992), 360 (384). Vgl. in diesem Zusammenhang auch Nelles, Deal - Bargaining im Strafprozess, in: Recht schafft Zukunft, hrsg. Däubler-Gmelin et.al. (2003), 226 (226 ff.).

12 Albrecht, KritV 1988, 182 (183 ff.); Hassemer, NStZ 1989, 553 (557 f.); Volk, JZ 1982, 85 (87).

13 a.a.O.

14 Schünemann, ZStW 52 (2002), 1 (26). Kritisch Hassemer, ZRP 1992, 378 (380).

15 Weigend, JZ 1990, 774 (780 f.).

16 Schünemann, FS Baumann (1992), 361 (367 f.); ders., ZStW 52 (2002), 1 (26); Weigend, JZ 1990, 774 (780 f.).

17 Löwe/Rosenberg - Rieß, Einl. Abschn. F Rn. 9 f.

18 Nicht von ungefähr wurde der erste Aufsatz, der sich eingehend mit der Thematik befasste, unter dem Pseudonym - es folgt Juristenhumor: - „Detlev Deal, Mauschelhausen« veröffentlicht, vgl. StV 1982, 545 (545 ff.)

19 Vgl. BGHSt 43, 195 (195 ff.).

20 Vgl. BGHSt 43, 195 (203 ff.). Vgl. zum Rechtsmittelverzicht, BGH, NJW 2005, 1440 (1440 ff.).

21 So die Formulierung von Weßlau, Konsensprinzip im Strafverfahren (2002), 144.

22 Krauß, FS Schaffstein (1975), 411 (413)

23 Volk, FS Salger (1994), 411 (412).

24 Klug, ZRP 1999, 288 (291); Schünemann, FS Pfeiffer 1988, 461 (474 f.).

25 Vgl. insoweit auch Klug, ZRP 1999, 288 (289 f.).

26 Vgl. Weigend ZStW 113 (2001), 271 (287).

27 Vgl. auch Schünemann, FS Pfeiffer (1988), 461 (483); ders., ZStW 114 (2002), 1 (20 ff.).

28 Vgl. auch Klug, ZRP 1999, 288 (291).

29 Hanack, StV 1987, 500 (501); Klug, ZRP 1999, 288 (291); Wolfslast, NStZ 1990, 409 (410).
30 Laut $\S 1$ BRAO ist der Rechtsanwalt ein unabhängiges Organ der Rechtspflege.

31 Vgl. insoweit Eser, ZStW 104 (1992), 360 (375).

32 Vgl. insoweit auch Eser, ZStW 104 (1992), 360 (371 f.); Löwe/Rosenberg-Rieß Einl. E Rn. 185 f.; Schünemann, FS Pfeiffer (1988), 461 (472); ders., ZStW 2002, 1 (25).

33 Rebmann, NStZ 1984, 241 (245 ff.); Schmitt, GA 2001, 411 (426).

34 Eser, ZStW 104 (1992), 360 (372).

35 Vgl. Hassemer, ZRP 1992, 378 (383).

36 Vgl. in diesem Zusammenhang auch Lüderssen, Abschaffen des Strafens (1995), 383 ff.; Prittwitz, StV 1991, 435 (437).

37 So die Formulierung von Schmidt-Hieber, NJW 1990, 1884 (1885 f.). Die These, dass der neuartige Verfahrenstyp vornehmlich in Wirtschaftsstrafverfahren praktiziert wird, wird nicht dadurch widerlegt, dass die Verfahrenspraxis auch außerhalb von Wirtschaftsstrafverfahren häufig auf die Opportunitätsvorschriften oder das Strafbefehlsverfahren zurückgreift. Anders als in Wirtschaftsstrafverfahren dürften die Gründe dort einerseits in der Erkenntnis um die sozial desintegrativen Folgen eines Strafverfahrens für den Angeklagten und andererseits in der bloß quantitativen Belastung des Strafjustizsystems liegen. Im Unterschied zu Wirtschaftsstrafverfahren dürften Beschuldigter und Verteidigung zudem auch weniger inhaltlichen Einfluss auf das Verfahrensergebnis haben, da es sich regelmäßig um Konstellationen handelt, in denen einem Sanktionsvorschlag von Staatsanwaltschaft und Gericht lediglich zugestimmt oder dieser akzeptiert wird.

38 Hassemer, JuS 1989, 890 (893 f.).

39 Schünemann, FS BGH (2002, 524 (541); Neumann, ZStW 101 (1989), 52 (70 f.).

40 Hassemer, JuS 1989, 890 (893 f.); Murmann, GA 2004, 65 (81 f.); Schünemann, FS Baumann (1992), 361 (376); ders., ZStW 114 (2002), 1 (28).

41 Vgl. Herrmann, JuS 1999, 1162 (1166 f.); Widmaier, StV 1986, 357 (359). Vgl. auch Frehsee, StV 1996, 222 (229).

42 Vgl. auch Jahn, GA 2004, 272 (287); Lüderssen, StV 1990, 415 (419); Schünemann, FS Pfeiffer (1988), 461 (483 f.), der entsprechende Überlegungen freilich auf die Bagatellkriminalität begrenzt sehen will.

43 Vgl. etwa Eser, ZStW 104 (1992), 360 (383); Lüderssen, StV 1990, 415 (419); Weigend, StV 2000, 63 (66).

44 Vgl. auch Bussmann/Lüdemann, Klassenjustiz oder Verfahrensökonomie (1995), 208 ff.; Schünemann, ZStW 114 (2002), 1 (28).

45 So bereits Volk, JZ 1982, 85 (87). Vgl. auch Eser, ZStW 104 (1992), 360 (395); Weigend, ZStW 113 (2001), 271 (302). 